University of Nebraska - Lincoln

DigitalCommons@University of Nebraska - Lincoln

2006

\title{
Sequence-Enabled Reassembly (SEER) Peptides for the Detection of DNA Sequences
}

\author{
Aik T. Ooi \\ University of Arizona \\ Cliff I. Stains \\ University of Nebraska-Lincoln, cstains2@unl.edu \\ Jason R. Porter \\ University of Arizona, \\ Indraneel Ghosh \\ University of Arizona, ghosh@email.arizona.edu \\ David J. Segal \\ University of California--Davis, djsegal@ucdavis.edu
}

Follow this and additional works at: https://digitalcommons.unl.edu/chemistrystains

Part of the Chemistry Commons

Ooi, Aik T.; Stains, Cliff I.; Porter, Jason R.; Ghosh, Indraneel; and Segal, David J., "Sequence-Enabled Reassembly (SEER) Peptides for the Detection of DNA Sequences" (2006). Cliff Stains Publications. 8. https://digitalcommons.unl.edu/chemistrystains/8

This Article is brought to you for free and open access by the Published Research - Department of Chemistry at DigitalCommons@University of Nebraska - Lincoln. It has been accepted for inclusion in Cliff Stains Publications by an authorized administrator of DigitalCommons@University of Nebraska - Lincoln. 
Published in Understanding Biology Using Peptides: American Peptide Symposia, 2006, Sylvie E. Blondelle, editor,

Volume 9, Part 3, pp. 214-215; doi: 10.1007/978-0-387-26575-9_91

Copyright (c) 2006 Springer Verlag. Used by permission.

\title{
Sequence-Enabled Reassembly (SEER) Peptides for the Detection of DNA Sequences
}

\author{
Aik T. Ooi, ${ }^{1}$ Cliff I. Stains, ${ }^{2}$ Jason R. Porter, ${ }^{2}$ Indraneel Ghosh, ${ }^{2}$ and David J. Segal ${ }^{1}$ \\ 1. Department of Pharmacology and Toxicology, University of Arizona, Tucson, AZ 85721, USA \\ 2. Department of Chemistry, University of Arizona, Tucson, AZ 85721, USA
}

\section{Introduction}

By combining custom zinc finger (ZF) DNA-binding technology [1,2] with protein fragment complementation [3], we have developed a technology, designated SEER (Sequence-Enabled Reassembly), that has the potential to "see" or detect genetic information within a living cell (Figure 1). These agents consist of two inactive parts of signal-generating peptides that have the ability to recognize specific DNA sequences. The two parts bind near each other in the presence of a user-defined DNA target site and generate a fluorescent signal. Two prototype SEER systems have been constructed, based on the reassembly of green fluorescent protein (SEERGFP) [4] and the enzyme $\beta$-lactamase (SEER-LAC). To our knowledge, these are the first examples of DNA-dependent reassembly of peptide fragments.

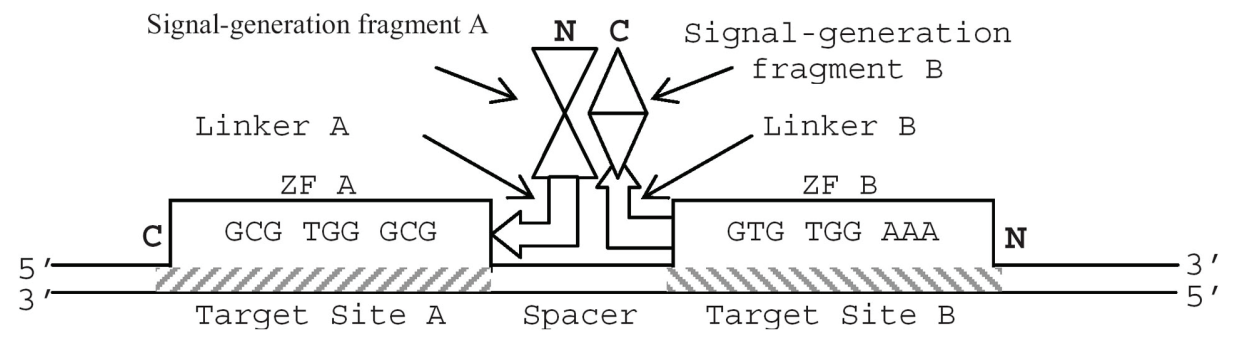

Figure 1. Schematic representation of a SEER system for sequence-specific detection of dsDNA.

\section{Results and Discussion}

Custom DNA-binding proteins: Since biologically relevant target sites cannot be chosen until the optimal spacer and orientation parameters are established, initial experiments employ designed target sites that are recognized by existing, well-characterized, ZF. Zif268 is a naturally-occurring 3-finger ZF that has been extensively studied structurally and biochemically. It binds the 9bp sequence 5'-GCGTGGGCG-3'. PBSII is a designed 3-finger ZF assembled from predefined modified ZF domains [1,2], which recognizes the sequence $5^{\prime}$-GTGTGGAAA-3'. Together, the two proteins recognize $18 \mathrm{bp}$ of DNA, a target site sufficiently large to be unique in the human genome.

SEER-GFP: Peptide constructs were designed such that the C-terminus of GFP fragment (1-157) was fused to the N-terminus of ZF Zif268 by means of a 15- residue linker and the N-terminus of GFP fragment (158-236) was fused to the C-terminus of ZF PBSII (Figure 2). Both proteins were separately purified under denaturing conditions. Equimolar mixtures $(15 \mu \mathrm{M}$ each) were refolded in the presence or absence of the target oligonucleotide $(4 \mu \mathrm{M})$. Fluorescence (at $505 \mathrm{~nm}$ ) was only observed for samples containing both halves of GFP-ZF fusions in the presence of target. No fluorescence was observed in the presence of the two half-sites alone or non-specific herring sperm DNA, demonstrating the specificity of reassembly. A 4-fold excess of target DNA strongly inhibited GFP reassembly, confirming the expectation that a high molar ratio of DNA:proteins localizes the two proteins to different oligonucleotides. 


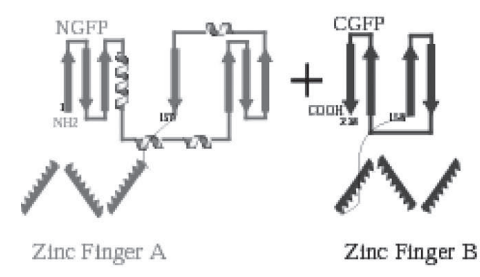

GFP-Zinc Finger Fragments

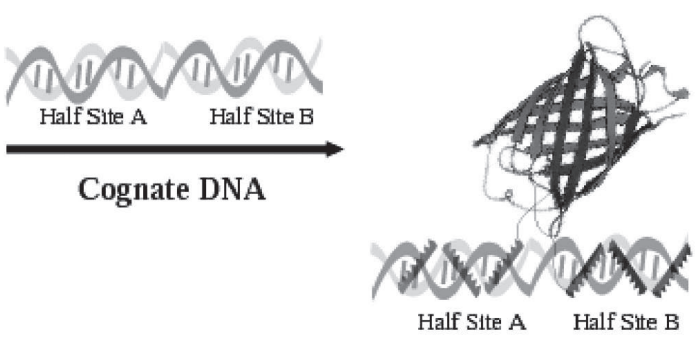

Fluorescent GFP bound to DNA

Figure 2. A cartoon illustrating the SEER-GFP.

SEER-LAC: A different approach is to use an enzyme, such as $\beta$-lactamase, to produce the signal instead of a single GFP molecule. Having an enzyme convert a substrate into an easily detectable product would amplify the signal. Genetic constructs were generated by standard cloning methods to code for the following two peptides: "LacA", an N-terminal zinc finger protein Zif268 linked by a 15aa (GlyGlyGlyGlySer) ${ }_{3}$ linker to residues 26-196 of E. coli TEM-1 $\beta$-lactamase, and "LacB", an N-terminal zinc finger protein PBSII linked by a 15aa linker to residues 198-290 of $\beta$-lactamase. LacA also contained a published M182T mutation in the $\beta$ - lactamase domain for greater stability [5]. The constructs were cloned into the HindII/NotI sites of the bacterial expression vector pMAL (New England Biolabs), which expressed proteins as C-terminal fusions with maltose binding protein (MBP). The MBP domain improves protein solubility, and allows one-step purification over amylose resin. SDS-PAGE analysis confirmed proteins at $>95 \%$ purity of approximately 72KD (LacA) and 65KD (LacB).

SEER should provide a sensitive yet inexpensive assay that may be useful as a clinical diagnostic agent. It addition to genomic rearrangements and telomere lengths, it could detect DNA accessibility, unusual structures and methylation, which are presently undetectable by similar methods. The most novel aspect of this method is its ability to recognize double-stranded DNA, presenting the possibility to report on genomic information within individual living cells, an ability not provided by any existing technology. The system could be reconfigured to kill cells through reactivation of a cytotoxic enzyme, producing sequence-dependent cell death. This research therefore impacts studies of disease detection and treatment.

\section{Acknowledgments}

This work was supported by the American Cancer Society (IRG7400125) and the NIH-NCI (SPORE in GI Cancer CA95060). C.I.S. received an NIH training grant.

\section{References}

1. Blancafort, P., Segal, D. J. and Barbas III, C. F. Mol. Pharmaco.l 66, 1361-1371 (2004).

2. Segal, D. J. Methods 26, 76-83 (2002).

3. Ghosh, I., Hamilton, A. D. and Regan, L. J. Am. Chem. Soc. 122, 5658-5659 (2000).

4. Stains, C. I., et al. J. Am. Chem. Soc. 127, 10782-10783 (2005).

5. Galarneau, A., et al. Nat. Biotechnol. 20, 619-622 (2002). 